\title{
Juristische Archivforschung und Rechtserkenntnis
}

\author{
Ruth Weber
}

\begin{abstract}
Der Zugang zu Rechtstexten erscheint selbstverständlich. Ein Gesetz, das nicht veröffentlicht ist, hat keine Geltung. Auch für Gerichtsentscheidungen gilt grundsätzlich eine Veröffentlichungspflicht. Doch was ist mit Materialien, die nicht auf Anhieb zu finden sind und für die es rechtliche Zugangshindernisse gibt? Die Entscheidungsakten des Bundesverfassungsgerichts sind nach sechzig Jahren im Bundesarchiv verfügbar; diejenigen des Gerichtshofs der Europäischen Union nach dreißig Jahren im Historischen Archiv der Europäischen Union. Rechtswissenschaftler:innen können damit neben historischen Gesetzgebungsmaterialien zunehmend auch Archivmaterialien gerichtlicher Entscheidungen, insbesondere Beratungsprotokolle, studieren. Können diese Materialien das gegenwärtige Verständnis der zur ständigen Rechtsprechung gewordenen Entscheidungen beeinflussen? Der Zugang zu rechtlichen Archivmaterialien soll binsichtlich seines Stellenwertes für den Rechtserkenntnisprozess reflektiert werden. Dafür ist es erforderlich zu untersuchen, was unter Archiven allgemein und im rechtswissenschaftlichen Kontext $z u$ verstehen ist. Sodann gilt es zu erklären, wie staatliche Archive rechtlich reguliert sind. Die Bedeutung der Forschung an gerichtlichen Archivmaterialien bildet den Fokus der Untersuchung des Verbältnisses von Archivforschung und Rechtserkenntnis. Die Grundthese des Textes lautet, dass juristische Archivforschung dazu geeignet ist, eine kontextualisierende Dogmatik zu operationalisieren. Zudem werden praktische Herausforderungen beim Umgang mit Archivmaterialien vorgestellt.
\end{abstract}

\section{Einleitung}

„W]as sucht [...] der Blick der Fotokamera, der direkt auf die endlosen, gleichförmigen Regalfluchten geht? [...] Innenansichten von menschenleeren Archivräumen mögen den Reiz eines verbotenen Bildes haben. Dem Geheimnis, das die Akten bergen, kommt die fotografische Nahaufnahme von Papierzeug [...] allerdings keine Spur näher. Akten wahren ibr Geheimnis auch vor zudringlichen Kamerablicken. [...] Doch gerade weil der Kamerablick die der Rechtsordnung zugewandte Seite der Akten, ihre Semantik, nicht erschließt, lenkt er das Augenmerk auf die Struktur des Archivs. Er registriert die Forma- 
tionen archivarischer Ordnung und man ist geneigt [...] in den Bildern von Regalreiben und Aktenstapeln Landschaften wiederzuerkennen, Bergschluchten, Steinbrüche, geologische Schichtungen von Endmoränen oder Ausblicke aufs freie Meer."

Die zitierten Worte stammen aus einem Aufsatz von Cornelia Vismann ${ }^{1}$, entstanden für einen Sammelband zu Geschichte, Theorie und Praxis des Wissens des Staates, in dem dazu passend ein Bildessay von Kai-Olaf Hesse erschien. ${ }^{2}$ Akten sind Alltagsgegenstände staatlicher Tätigkeit. ${ }^{3}$ Werden sie nicht mehr gebraucht, wandern sie ins Archiv. So einfach das klingt, so einfach ist es nicht: Denn wer entscheidet, welche Akten ins Archiv gelangen? Was geschieht mit den Akten, wenn sie einmal im Archiv angekommen sind? Wer hat dann noch Zugang zu den Akten und dem darin enthaltenen Wissen? Was bedeutet es schließlich für den Rechtserkenntnisprozess, Zugang zu archivalischem Wissen zu haben?

All diese Fragen lassen sich stellen, wenn man sich mit staatlichen Archiven als hoheitlichen Informationsspeichern beschäftigt. Um zu klären, was unter "Archiv“ allgemein zu verstehen ist, ist zunächst der Begriff als solcher zu klären. Im zweiten Teil soll die rechtliche Regulierung des Archivs näher erläutert werden. Drittens soll der Leitfrage nach dem Verhältnis von juristischer Archivforschung und Rechtserkenntnis nachgegangen werden, um zuletzt mit einigen praktischen Überlegungen zur Archivforschung zu enden.

1 Vismann, Was weiß der Staat noch?, in: Collin/Horstmann (Hrsg.), Das Wissen des Staates. Geschichte, Theorie und Praxis, 2004, S. 41 (42).

2 Die Photographien wurden in der Präsentation begleitend zum Text von Vismann gezeigt. S. Hesse, Zur Phänomenologie staatlichen Wissens, in: Collin/Horstmann (Hrsg.), Das Wissen des Staates (Fn. 1), S. 47.

3 Die Materialität der Akten beschreibt auch Latour in La fabrique du droit. Une ethnographie du Conseil d'État, 2004, S. 83-84: „Quoi de plus grisâtre, de plus poussiéreux, de plus méprisable que des piles de dossiers ? [...] [I]l existe un traceur qui organise toute l'activité du Conseil, [...] c'est le dossier. [...] Oui, commençons le droit par le commencement, c'est-à-dire par les tampons, les élastiques, les trombones et autre agrafeuses [...]. Les juristes parlent toujours des textes, mais rarement de leur matérialité.“ 


\section{I. „Archiv“}

\section{Etymologie}

In seiner Materialität besteht das „Archiv“ aus den eingangs betrachteten Akten und übrigen Materialien, die Archivar:innen in einem physischen Speicher aufbewahren und verwalten. ${ }^{4}$ Die auf den Aufbewahrungsort rekurrierende, institutionelle Verwendungsweise des Begriffs knüpft an die Ursprünge des Wortes an. ${ }^{5}$ Das lateinische archium ${ }^{6}$ geht auf das griechi-

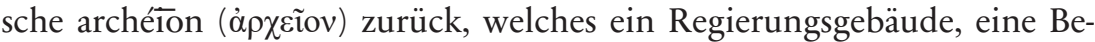
hörde oder ein Amt bezeichnete. ${ }^{7}$ Archéion leitet sich seinerseits von arché

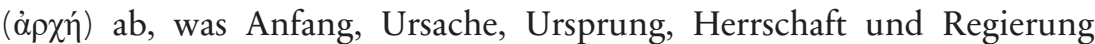
bedeutet. ${ }^{8}$ Die Etymologie zeigt, dass der Bezug auf ein Gebäude oder Amt eine abgeleitete Form eines abstrakten Begriffs ist. In der Begriffsvariante der Herrschaft ist das Archiv „eng mit der Machtgeschichte verknüpft“"

4 Vismann beschreibt das Archiv „zunächst [als] nichts anderes als eine Verwahranstalt für Akten“ (Vismann, Akten. Medientechnik und Recht, 3. Aufl. 2011, S. 177); S. auch Wirth, Archiv, in: Roesler/Stiegler (Hrsg.), Grundbegriffe der Medientheorie, 2005, S. 17.

5 Stichwort „Archiv“, in: Pfeifer et al., Etymologisches Wörterbuch des Deutschen (1993), digitalisierte und von Wolfgang Pfeifer überarbeitete Version im Digitalen Wörterbuch der deutschen Sprache, <https://www.dwds.de/wb/etymwb/Archiv>(letzter Aufruf am 7.2.2021).

6 Im antiken Rom wurde das Archiv „zum Speichermedium des römischen Rechtssystems“ (Wirth, Archiv (Fn. 4), S. 17 (18)). Das „Aerarium“ als „materiale[s] Fundament der römischen Verwaltung“ „ist ein Depot, welches einen unverlierbaren Thesaurus beherbergt und hütet“, das „allein in der Geste der Bezugnahme, aufgrund einer Verweisungsmöglichkeit" existiert (Vismann, Akten (Fn. 4), S.91). Archivierbare Akten sind demnach für Vismann "das Kapital des Rechts“ (Vismann, Akten (Fn. 4), S. 92).

7 Stichwort „Archiv“, in Pfeifer et al., Etymologisches Wörterbuch (Fn. 5). Derrida befasst sich mit den Archonten, die für ihn die „Bewahrer“ des Archivs sind und „nicht nur die physische Sicherheit des Depots und des Trägers“ sicherstellen, sondern darüber hinaus die „Macht“ haben, „die Archive zu interpretieren“ und so „das Gesetz“ „behaupten“ (Derrida, Dem Archiv verschrieben. Eine Freudsche Impression, 1997, S. 11; zur Etymologie s. S. 9-13).

8 Stichwort „Archiv“, in Pfeifer et al., Etymologisches Wörterbuch (Fn. 5).

9 Lepper, Philologie zur Einführung, 2012, S. 73. Zur "Idee des Archivs“ in den verschiedenen Varianten auch Lepper/Raulff, Erfindung des Archivs, in: Lepper/Raulff (Hrsg.), Handbuch Archiv. Geschichte, Aufgaben, Perspektiven, 2016, S. 1; Schenk, Kleine Theorie des Archivs, 2008, S. 60-64. Zu den Zusammenhängen von Archiven und Herrschaft aus der archivarischen Praxis s. Murken (Hrsg.), Archive und Herrschaft. Referate des 72. Deutschen Archivtages 2001 in Cottbus, 2002, Der Ar- 
Unter Rückgriff auf die Ursprünge des Begriffs „Archiv“ ergibt sich ein deutlich weiteres Begriffsverständnis, das man auch als metaphorisch bezeichnen könnte. Beispielhaft dafür steht die Verwendungsweise bei Jacques Derrida und Michel Foucault, für welche das "Archiv“ nicht mehr an seine Materialität gebunden ist. In „Dem Archiv verschrieben“ ist es für Derrida vielmehr eine politische Kategorie; politische Macht ist für ihn verknüpft mit Kontrolle über das Archiv und das Gedächtnis. ${ }^{10}$ Auch Foucault verwendet den Begriff des "Archivs" in "Archäologie des Wissens" metaphorisch und als Ausdruck eines gesellschaftlichen Gedächtnisses. ${ }^{11}$

\section{Archivzeitschriften}

Im rechtswissenschaftlichen Kontext begegnet einem der Begriff „Archiv“ auch im Titel der sogenannten „Archivzeitschriften“. Die Geschichte dieser Bezeichnung bestätigt die Mehrdeutigkeit des Begriffs.

Als die ersten sich als „Archiv“ bezeichnenden Zeitschriften im ausgehenden 17. Jahrhundert entstanden, war deren Ziel noch hauptsächlich, sonst unzugängliches Material offenzulegen. ${ }^{12}$ Bereits bei Gründung des Archivs für die civilistische Praxis (AcP) Anfang des 19. Jahrhunderts galt die Bezeichnung jedoch als altmodisch. ${ }^{13}$ Bei der Entstehung des

chivar, Beiband 7. Völkerrechtlich stellt sich das Problem des Umgangs mit Archiven bei einem Herrschaftswechsel, s. Fitschen, Das rechtliche Schicksal von staatlichen Akten und Archiven bei einem Wechsel der Herrschaft über Staatsgebiet, 2004.

10 Derrida, Dem Archiv verschrieben (Fn. 7), Assmann, Erinnerungsräume. Formen und Wandlungen des kulturellen Gedächtnisses, 5. Aufl. 2010, S. 343-344; Ernst, Das Rumoren der Archive. Ordnung aus Unordnung, 2002.

11 Foucault beschreibt das Archiv als Möglichkeiten von Aussagen, „das Gesetz dessen, was gesagt werden kann“ (S. 187), welche ein "System der Diskursivität“ (ebd.) bilden, s. Foucault, Archäologie des Wissens, 4. Aufl. 1990. Weiterführend Bergermann, Linkspeicher Google. Zum Verhältnis von PageRank und Archäologie des Wissens, in: Weitin/Wolf (Hrsg.), Gewalt der Archive. Studien zur Kulturgeschichte der Wissensspeicherung, 2012, S. 371.

12 Dann, Die Zeitschriften im Rahmen der deutschen Aufklärungsgesellschaft, in: Stolleis (Hrsg.), Juristische Zeitschriften. Die neuen Medien des 18.-20. Jahrhunderts, 1999, S. 1 (3-4), zum Zusammenhang mit der Öffnung und Demokratisierung des Diskurses s. ebd. S. 9.

13 Übersicht zu weiteren in diesem Zeitraum entstehenden rechtswissenschaftlichen Archivzeitschriften bei Rückert, Geschichtlich, praktisch, deutsch. Die „Zeitschrift für geschichtliche Rechtswissenschaft“ (1815-1859), das „Archiv für die civilistische Praxis“ (1818-1867) und die „Zeitschrift für deutsches Recht und deutsche 
Archivs des öffentlichen Rechts (AöR) am Ende des 19. Jahrhunderts diskutierte man zwar über die Kennzeichnung „für öffentliches Recht"; die Bezeichnung als „Archiv“ blieb demgegenüber unreflektiert. ${ }^{14}$ Jedenfalls gilt seit der Gründungszeit dieser auch noch heute besonders relevanten Zeitschriften, dass diese „Kontinuität für einen Sammelplatz“ boten, ohne einen „spezifischen Akzent auf Praxis oder Fälle oder Wissenschaft" zu setzen. ${ }^{15}$ Dem eigenen Anspruch nach bilden Archivzeitschriften einen Speicher relevanten Wissens für ihr jeweiliges Fach. Gleichzeitig haben sie ihren Ursprung im 19. Jahrhundert, welches auch als Jahrhundert der (Staats-)Archive gekennzeichnet wird; als „die Epoche, als der Staat sich überall der Erinnerung bemächtigte“.${ }^{16}$

\section{Recht im Archiv}

In einem ähnlichen Zeitraum wie die Archivzeitschriften entstanden auch die ersten neuzeitlichen rechtlichen Regulierungen von Archiven. Bereits

Rechtswissenschaft“ (1839-1861), in: Stolleis (Hrsg.), Juristische Zeitschriften (Fn. 12), S. 107 (154).

14 Zur Geschichte des 1885 gegründeten AöR s. Doerfert, Das Archiv des öffentlichen Rechts 1885-1918, 1993, insb. zur Namensfrage S. 34-40. S. auch Heyen, Profile der deutschen und französischen Verwaltungsrechtswissenschaft 1880-1914, 1994, S. 21-53, für einen Vergleich mit anderen Zeitschriften in der Gründungsphase ebd., S. 55-71. Für einen Vergleich mit dem 1892 gegründeten Verwaltungsarchiv s. Doerfert, Die Zeitschriften des öffentlichen Rechts 1848-1933, in: Stolleis (Hrsg.), Juristische Zeitschriften (Fn. 12), S. 421 (436-440).

15 Rückert, Geschichtlich, praktisch, deutsch (Fn. 13), S. 107 (154). Die Idee war bereits bei Gründung des AcP nicht neu. Das 1806 gegründete „Critische Archiv der neuesten juridischen Litteratur und Rechtspflege in Teutschland" machte sich beispielsweise zur Aufgabe, die „Idee des Ganzen unserer Rechtsverfassung und Rechtslehre in sich [zu] tragen“" (zitiert nach Klippel, Die juristischen Zeitschriften im Übergang vom 18. zum 19. Jahrhundert, in: Stolleis (Hrsg.), Juristische Zeitschriften (Fn. 12), S. 15 (35)). Auch für andere Fächer gelten die Überlegungen zur Bezeichnung als Archiv, s. bspw. für das 1904 entstehende, unter anderem von Max Weber verantwortete „Archiv für Sozialwissenschaften und Sozialpolitik“ Duve, Von „Stoffhubern“ und „Sinnhubern“. Die wissenschaftstheoretische Grundlagendebatte um 1900 und die Entstehung politikwissenschaftlicher Zeitschriften, in: Stolleis (Hrsg.), Juristische Zeitschriften (Fn. 12), S. 449 (472-477).

16 Viele Staatsarchive wurden „als zentralisierende Lagerstätten der Überreste von Verwaltungshandeln“ gegründet, s. Osterhammel, Die Verwandlung der Welt. Eine Geschiche des 19. Jahrhunderts, 3. Aufl. 2020, S. 32-33, beide Zitate S. 32. Combe spricht von Archiven als „une institution du XIX siècle“, s. Combe, Archives Interdites, Les peurs françaises face à l'histoire contemporaine, 1994, S. 49-75. 
1766 sah das schwedische Gesetz über die Pressefreiheit („Tryckfrihetsförordning“") ein Recht auf freien Zugang zu Archiven vor. ${ }^{17}$ Während der Französischen Revolution ${ }^{18}$ wurde der unbeschränkte „Archivzugang“ gleichsam „zum Menschenrecht erklärt“19. Das verborgene Wissen des Staates sollte nun zugänglich sein. ${ }^{20}$ Preußen öffnete erst hundert Jahre später sein Geheimes Staatsarchiv. ${ }^{21}$ Weitere hundert Jahre sollte es dauern, bis das Bundesarchivgesetz (BArchG) 1988 in Kraft trat. ${ }^{22}$ Die Geset-

17 Die „Tryckfrihetsförordning“ ist noch heute Bestandteil der schwedischen Verfassung und gilt heute in der Fassung von 1949. Sie gewährt zum einen umfangreich Presse- und Meinungsfreiheit und zum anderen den Zugang zu allen staatlichen Dokumenten („Offentlighetsprincipen") mit nur wenigen geregelten Ausnahmen. In der ursprünglichen Fassung von 1766 heißt es in $\$ 10$ : „And to that end free access should be allowed to all archives, for the purpose of copying such documents in loco or obtaining certified copies of them", s. His Majesty's Gracious Ordinance Relating to Freedom of Writing and of the Press (1766), translated by Hogg, in: Mustonen (Hrsg.), The World's First Freedom of Information Act, Anders Chydenius' Legacy Today, 2006. S. 15 (abrufbar unter: https://www.chydeniu s.net/tiedostot/worlds_first_foia.pdf). Zum Gesetz von 1776 s. Nordin, Journal of International Media \& Entertainment Law, 2017-2018 7 (2), 137.

18 Die Gesetze aus der Zeit der Revolution galten in Frankreich bis 1979, s. Ducrot, Die neue französische Archivgesetzgebung, Der Archivar 34 (1981), 475 sowie Combe, Archives Interdits (Fn. 16), S. 79-102; Kamecke, Das Revolutionsarchiv von 1789 und das Problem der Geschichtsschreibung, in: Weitin/Wolf (Hrsg.), Gewalt der Archive (Fn. 11), S. 141. S. von der Französischen Revolution ausgehend zur „Archiv-Rechtsgeschichte“ als „Zugangsgeschichte rekonstruiert“ Kaiser, Archiv und Recht, in: Lepper/Raulff (Hrsg.), Handbuch Archiv (Fn. 9), S. 107 (108-111).

19 Vismann, Akten (Fn. 4), S. 249. S. auch Brenneke, Archivkunde. Ein Beitrag zur Theorie und Geschichte des europäischen Archivwesens, 1953: „Erklärung der archivalischen Menschenrechte““ (S.182). Die Erklärung der Menschen- und Bürgerrechte von 1789 enthält kein explizites Recht auf Zugang zu Archiven.

20 Zum Zusammenhang mit staatlichem Wissen s. Vismann, Akten (Fn. 4), S. 242-252: Das Archiv ist nach Vismann „Konstituens für die Nation“ (S. 242). Sie kontrastiert den „Furor der Aktenzerstörung“ (S. 242) mit dem revolutionären Gedanken, „der Nachwelt einen Begriff davon zu geben, welche Schreckensherrschaft sie [die Franzosen] beendet haben“ (S. 243). S. dazu auch Assmann, Erinnerungsräume (Fn. 10), S. 344-345.

21 S. dazu sowie zu den Regelungen vor Kodifikation des BArchG Lepper, DVBl 1963, 315. Für Vismann wird das Staatsarchiv „zum Machtdispositiv des preußischen Staats“ (Vismann, Akten (Fn. 4), S. 246).

22 BT-Drucks. 11/498. S. dazu Wyduckel, DVBl 1989, 327. Daneben bestehen zahlreiche landes- sowie kommunalrechtliche Regelungen. Beispielhaft zu NRW Oebbecke/Nienkemper DVBl 2004, 1509. 
zesbegründung bezeichnet das Archiv als „Gedächtnis des Staates“, ein Ausdruck, der die Wissensdimension veranschaulicht. ${ }^{23}$

Das BArchG soll im Folgenden als Beispiel dienen, einen Blick auf das Archivrecht ${ }^{24}$ zu werfen. Dabei stellen sich zwei wesentliche Fragen, zum einen danach, welche Akten überhaupt im Archiv aufbewahrt werden, zum anderen, wer Zugang zu den Akten hat.

\section{Archivarische Bewertung: Aufnahme in die Bestände eines Archivs}

Bevor die Akte im Archiv angelangt, ist die jeweilige Behörde verpflichtet, diese anzubieten und abzugeben. ${ }^{25}$ Archivar:innen kommt im Anschluss die Bewertungsentscheidung zur Archivfähigkeit zu. Es muss sich nach der Terminologie des Gesetzes um „Unterlagen von bleibendem Wert“ ${ }^{\text {"26 }}$ handeln. Bleibenden Wert haben nach der Legaldefinition ${ }^{27} \mathrm{u}$. a. auch Unterlagen, die aufgrund ihres rechtlichen Inhalts Bedeutung für die Rechtsprechung haben, was als Hinweis darauf gedeutet werden könnte, dass Archivmaterialien für die Rechtsanwendung von Relevanz sind.

Verwaltungsrechtlich erfolgt an dieser Stelle ein Umwidmungsakt: Akten der laufenden Verwaltung werden zu Kulturgut des Bundes; die Akte wird von der öffentlichen Sache im Verwaltungsgebrauch zu einer solchen im Gemeingebrauch. ${ }^{28}$

23 BT-Drucks. 11/498, S. 7: „Gedächtnis des Staates“. Diese Bezeichnung für das Archiv findet man häufig, z. B. auch bei Ladeur, Die Kommunikationsinfrastruktur der Verwaltung, in: Hoffmann-Riem/Schmidt-Aßmann/Voßkuhle (Hrsg.), GVwR II, 2. Aufl. 2012, $\$ 21$ Rn. 35 und bei Assmann, Erinnerungsräume (Fn. 10): „Archiv als Gedächtnis der Herrschaft“ (S. 343), als „institutionalisiertes Gedächtnis der Polis, des Staates, der Nation, der Gesellschaft" (S. 345).

24 Die Bezeichnung dieses Rechtsgebiets als "Archivrecht" scheint sich durchgesetzt zu haben. Günther bevorzugt die Bezeichnung als „Archivverwaltungsrecht“, s. Günther, Archive und Verwaltung oder: Über die Grenzen des Archivrechts, in: Becker et al. (Hrsg.), Archiv - Recht - Geschichte. Festschrift für Rainer Polley, Veröffentlichungen der Archivschule Marburg Nr. 59, 2014, S. 195.

$25 \$ 5$ BArchG.

26 S. $\mathbb{1} 1$ Nr. 2 BArchG für die Legaldefinition von Archivgut, $\mathbb{1} 1$ Nr. 9 BArchG für die Legaldefinition von Unterlagen und $\$ 1$ Nr. 10 BArchG für die Legaldefinition von Unterlagen von bleibendem Wert.

$27 \$ 1$ Nr. 10 a) cc) BArchG.

28 Partsch/Koschmieder, NJW 2017, 3416 (3417-3418); Olbertz, Das Bundesarchivgesetz im Kontext der neuen Gesetzgebung zum Informationszugang in der Verwaltung, in: Menne-Haritz/Hofmann (Hrsg.), Archive im Kontext. Öffnen, Erhalten und Sichern von Archivgut in Zeiten des Umbruchs. Festschrift für Hartmut 


\section{Der Zugang zum Archiv: Nutzungsanspruch}

Nachdem sich die Akte also nun im Archiv befindet, stellt sich die zweite rechtlich relevante Frage: Wer hat nun Zugang ${ }^{29}$ zum Archiv? Nach $\$ 10$ Abs. 1 BArchG steht das Recht, Archivgut des Bundes zu nutzen, grundsätzlich jeder Person voraussetzungslos zu. ${ }^{30}$ Dies scheint auf den ersten Blick eine einfache Regel zu sein, die aber viele faktische Hürden ${ }^{31}$ und rechtliche Ausnahmen kennt.

Die wichtigste rechtliche Ausnahme sind die Schutzfristen, die allgemein 30 Jahre betragen. ${ }^{32}$ Diese Regelung ist umstritten: Gerade aufgrund der Wertungswidersprüche mit dem Informationsfreiheitsrecht, das einen zeitlich nicht begrenzten Anspruch auf Zugang zu amtlichen Informationen normiert, wird gefordert, die archivrechtliche Schutzfrist abzuschaffen. ${ }^{33}$ Die Ansprüche des BArchG stellen nach der Rechtsprechung Son-

Weber zum 65. Geburtstag, 2010, S. 139 (139, 152), Fn. 64 m. w. N.; Becker/Oldenhage, HK-BArchG, 2006, $\$ 2$ Rn. 18.

29 Archivterminologisch wäre wohl der Begriff der „Zugänglichkeit“ dem Begriff des „Zugangs“ vorzuziehen, s. Menne-Haritz, Schlüsselbegriffe der Archivterminologie. Lehrmaterialien für das Fach Archivwissenschaft, Archivschule Marburg 1992, S. 58.

30 Neben dem Nutzungsanspruch gegen das Archiv selbst besteht nach $₫ 11$ Abs. 6 BArchG ein Nutzungsanspruch gegen all die Behörden, welche Unterlagen, die älter als 30 Jahre sind, behalten, gleich ob sie eine Anbietungspflicht haben oder nicht. In den landesrechtlichen Vorschriften wird teils ein berechtigtes Interesse gefordert, s. Collin, Die archivrechtliche Regulierung des Zugangs zu öffentlichen Informationen, in: Dreier/Fischer/van Raay/Spiecker gen. Döhmann (Hrsg.), Informationen der öffentlichen Hand - Zugang und Nutzung, 2016, S. 209 (213-214), insb. Fn. 16 und 17.

31 Collin, Die archivrechtliche Regulierung (Fn. 30), S. 209 (214). Solche faktischen Hürden sind etwa der Umgang mit den Findmitteln sowie der Erschließungszustand der Bestände, s. auch Abschnitt IV.1.

$32 \$ 11$ Abs. 1 BArchG. Für einen Überblick zu den landesrechtlichen Vorschriften, die teilweise eine Regelschutzfrist von nur 10 Jahren vorsehen s. Dittrich, ZRP 2017, 183. Dittrich spricht sich für eine ,generelle Liberalisierung der Schutzfristenregelung“ (S. 185) aus.

33 Olbertz, Das Bundesarchivgesetz (Fn. 28), S. 139 (146); Manegold, Archivrecht. Die Archivierungspflicht öffentlicher Stellen und das Archivzugangsrecht des historischen Forschers im Licht der Forschungsfreiheitsverbürgung des Art. 5 Abs. 3 GG, 2002. S. 269; kritisch auch Martin-Weber, Informationsfreiheitsgesetz des Bundes (IFG) und Bundesarchivgesetz, in: Rehm/Bickhoff (Hrsg.), Rechtsfragen der Nutzung von Archivgut, 2010, S. 17; Collin, Die archivrechtliche Regulierung (Fn. 30), S. 209 (214-218); Schoch, Die Verwaltung 2006, 463 (465); Kaiser, Archiv und Recht (Fn. 18), S. 107 (111). 
dervorschriften nach $\mathbb{1} 1$ Abs. 3 IFG dar, die den Anwendungsbereich des IFG ausschließen. ${ }^{34} \mathbb{} \$ 1$ Abs. 5 Nr. 2 BArchG regelt das Verhältnis zwischen IFG und BArchG grundsätzlich. Danach sind die Schutzfristen nicht anzuwenden, soweit das Archivgut aus Unterlagen besteht, die vor der Übergabe an das Bundesarchiv bereits einem Informationszugang nach einem Informationszugangsgesetz offen gestanden haben. Das BArchG normiert zudem eine ganze Bandbreite an Einschränkungs- und Versagungsgründen, etwa das Wohl der Bundesrepublik, schutzwürdige Interessen Betroffener, Geheimhaltungspflichten und das Entstehen unverhältnismäßigen Verwaltungsaufwands. ${ }^{35}$

Gegen eine ablehnende Entscheidung kann Rechtsschutz vor dem Verwaltungsgericht gesucht werden. ${ }^{36}$ Dabei spielen insbesondere auch die konfligierenden verfassungsrechtlichen Positionen eine Rolle, insbesondere die in Art. 5 GG verankerten Rechte auf Informations- sowie Forschungs- und Wissenschaftsfreiheit. Dem stehen der staatliche Geheimnisschutz sowie die Persönlichkeitsrechte, insbesondere das Recht auf informationelle Selbstbestimmung, gegenüber. ${ }^{37}$

34 S. zu $\$ 11$ Abs. 6 BArchG OVG Berlin-Brandenburg, Urteil vom 7.5.2020 - OVG 12 B 4.19, Rn. 23; zu $₫ 5$ BArchG a. F. BVerwG, Urteil vom 17.3.2016 - BVerwG 7 C 2.15, BVerwGE 154, 231, Rn. 42; beide Entscheidungen mit einem recht spärlichen Verweis auf die Gesetzesbegründung (BT-Drucks. 15/4493, S. 8), die diesbezüglich jedoch keine klare Aussage trifft.

$35 \rrbracket 13$ Abs. 1 Nr. 1-3 und Abs. 2 Nr. 2 BArchG. Zur Rechtsprechung und zu den Regelungen im Einzelnen s. Partsch/Koschmieder, NJW 2017, 3416.

36 Zum Rechtsschutz s. Manegold, Archivrecht (Fn. 33), S. 356-359. Für beispielhafte jüngere Entscheidungen s. OVG Münster, Urteil vom 5.7.2018 - 15 A 2147/13 (Verpflichtungsklage nach Verkürzung der Sperrfrist nach $\$ 11$ BArchG n. F.); OVG Münster, Urteil vom 15.5.2018 - 15 A 25/17 (Versagungsgründe nach $\$ 5$ Abs. 6 BArchG a. F.); BVerwG, Beschluss vom 27.5.2013 - BVerwG 7 B 43.12 (Begriff des Archivguts/Zugang zu amtlichen Dokumenten im Privatbesitz). Die darauf eingelegte Verfassungsbeschwerde hatte keinen Erfolg, da sie das BVerfG aus Subsidiaritätsgründen als unzulässig verwarf, s. BVerfG, Beschluss vom 20.6.2017 - 1 BvR 1978/13, BVerfGE 145, 365; BVerwG, Urteil vom 11.12.2019 - BVerwG 6 C 21.18, BVerwGE 167, 173 (Schutzfrist nach $\$ 11$ BArchG bei Personenakten des Bundesamtes für Verfassungsschutz).

37 Ein unmittelbarer Nutzungsanspruch ergibt sich weder aus Art. 5 Abs. 3 noch aus Art. 5 Abs. 1 S. 1 GG. Allein aus der Pressefreiheit (Art. 5 Abs. 1 S. 2 GG) ergibt sich ein verfassungsunmittelbarer Auskunftsanspruch für Presseangehörige (BVerfG, Beschluss vom 14.9.2015 - 1 BvR 857/15, BVerfG NJW 2015, 3708, Rn. 15 ff.). 


\section{Rechtserkenntnis und Archiv}

Das Recht regelt den Zugang zum Archiv. Hat man einmal Zugang zu archivalischem Material, stellt sich die Frage, welches Potential juristische Archivforschung für Rechtserkenntnis und Rechtsdogmatik birgt. Die folgenden Ausführungen konzentrieren sich deshalb auf gerichtliches Archivmaterial, insbesondere das des Bundesverfassungsgerichts.

\section{Das Archivmaterial des Bundesverfassungsgerichts}

Das Bundesverfassungsgericht zum Anschauungsobjekt zu machen, ist angesichts seiner herausragenden Stellung im deutschen Rechtssystem und seines spezifischen Begründungsstils besonders interessant. ${ }^{38}$ Der 2013 neu gefasste ${ }^{39} \$ 35$ b Abs. 5 BVerfGG trifft eine eigene Schutzfristenregelung für das Gericht: Für die Einsicht in die Verfahrensakten gilt die auch im BArchG gewöhnliche Frist von 30 Jahren. Demgegenüber besteht für besonders sensible Dokumente eine 60 -jährige Frist ${ }^{40}$, wozu vor allem Voten und Notizen aus dem Beratungszimmer zählen. Die längere Frist betrifft also diejenigen Akten, die es ermöglichen können, den internen Entscheidungsfindungsprozess besser nachzuvollziehen. Mit dem in diesem Jahr 70-jährigen Bestehen des Gerichts ist eine Auswertung des Archivmaterials für die Entscheidungen der 1950er Jahre möglich. Diese sind besonders interessant, da sie einerseits in einer die Wirkmacht des Bundesverfassungsgerichts prägenden Phase entstanden ${ }^{41}$ und andererseits die Veröffentli-

38 Vergleichend zum Begründungsstil von BVerfG und Conseil constitutionnel s. Weber, Der Begründungsstil von Conseil constitutionnel und Bundesverfassungsgericht. Eine vergleichende Analyse der Spruchpraxis, 2019.

39 Gesetz zur Änderung des BVerfGG vom 29.8.2013, BGBl. 2013 I, S. 3463; Gesetzesentwurf BT-Drucks. 17/13469. Zur Gesetzesänderung und zum Erschließungszustand s. Meinel/Kram, JZ 2014, 913; Hollmann, Die Akten des Bundesverfassungsgerichts im Bundesarchiv. Zur Geschichte des Bestands B 237 und seiner archivischen Erschließung, in: Meinel (Hrsg.), Verfassungsgerichtsbarkeit in der Bonner Republik. Aspekte einer Geschichte des Bundesverfassungsgerichts, 2019, S. 97 (107-108).

40 Die 60-jährige Frist gilt auch für andere Unterlagen mit Geheimhaltungspflichten im Bereich des Steuer-, Sozial- und Bankrechts, s. $\$ 11$ Abs. 3 i. V. m. $\$ 6$ Abs. 1 S. 1, Abs. 4 BArchG.

$41 \mathrm{Wahl}$, Das Bundesverfassungsgericht in der Gründungsphase. Entwicklungsgeschichte der Institution und der Rechtsprechung, in: Meinel (Hrsg.), Verfassungsgerichtsbarkeit in der Bonner Republik (Fn. 39), S. 27 (33-36); Collings, Democra- 
chung von Sondervoten in diesem ersten Jahrzehnt noch nicht erlaubt war. ${ }^{42}$

Angesichts dieser Ausgangslage entstanden in den letzten Jahren eine ganze Reihe von Auswertungen des Bundesarchiv-Bestandes „B 237“, in dem sich die Materialien zum Bundesverfassungsgericht befinden. ${ }^{43}$ Die Beiträge rekonstruieren beispielsweise die „Vergangenheitsbewältigung“ im „Beamtenurteil“44, die Entstehung der Grundrechtsdogmatik zu Art. 2 Abs. 1 GG im „Elfes-Urteil“ 45 sowie das bundesverfassungsgerichtliche Verständnis des Verhältnisses von Exekutive und Legislative im „Redezeit-Urteil" 46 . Eindrucksvoll wird so deutlich, wie um die Entscheidungen in den Anfangsjahren gerungen wurde und welche Rolle Berichterstatter:innen und Richterpersönlichkeiten hatten. Es zeigt sich außerdem, dass das Bundesverfassungsgericht nicht in einem institutionellen Vakuum handelte, sondern insbesondere das Verhältnis zum Bundesgerichtshof, aber auch zu den Bundesorganen, prägend war. Die Archivalien zeigen, dass den Urtei-

cy's Guardians. A History of the German Federal Constiutional Court, 1951-2001, 2015; Hailbronner, Traditions and Transformations. The Rise of German Constitutionalism, 2015; Gaillet, La Cour constitutionnelle fédérale allemande. Reconstruire une démocratie par le droit (1945-1961), i. E., 2021.

42 Sondervoten sind nach $₫ 30$ Abs. 2 S. 1 BVerfGG seit 1971 zulässig, Gesetz zur Änderung des BVerfGG vom 21.12.1970, BGBl. 1970 I, S. 1765.

43 Allgemein zum Bundesarchiv s. Weber, Archivische Grundversorgung - die Staatsarchive, in: Lepper/Raulff (Hrsg.), Handbuch Archiv (Fn.9), S. 90 (94-95) und insb. zu den Akten des BVerfG Pawelletz, Der Zugang zu den Akten des Bundesverfassungsgerichts aus archivrechtlicher Sicht, in: Deiseroth/Weinke (Hrsg.), Zwischen Aufarbeitung und Geheimhaltung. Justiz- und Behördenakten in der Zeitgeschichtsforschung, 2021, S. 147. Zur Erforschung der Archivmaterialien s. insb. den Sammelband Meinel (Hrsg.), Verfassungsgerichtsbarkeit in der Bonner Republik (Fn. 39); Darnstädt, Verschlusssache Karlsruhe. Die internen Akten des Bundesverfassungsgerichts, 2. Aufl. 2019; Deiseroth/Weinke (Hrsg.), Zwischen Aufarbeitung und Geheimhaltung (Fn. 43), S. 101.

44 BVerfG, Urteil vom 17.12.1953 - 1 BvR 147/52, BVerfGE 3, 58 bei Meinel/Kram, JZ 2014, 913 (919-920) und Roßbach, Weichenstellung und Vergangenheitsbewältigung im Öffentlichen Dienst. Das Urteil zum G 131, in: Meinel (Hrsg.), Verfassungsgerichtsbarkeit in der Bonner Republik (Fn. 39), S. 229.

45 BVerfG, Urteil vom 16.1.1957 - 1 BvR 253/56, BVerfGE 6, 32 bei Grimm, Das Elfes-Urteil, in: Meinel (Hrsg.), Verfassungsgerichtsbarkeit in der Bonner Republik (Fn. 39), S. 155 und Darnstädt, NJW 2019, 1580 (1581-1584).

46 BVerfG, Urteil vom 14.7.1959 - 2 BvE 2/58, BVerfGE 10, 4 bei Cancik, Parlamentarismus vor dem Bundesverfassungsgericht. Das Redezeiturteil und die Erfassung der Verfassungswirklichkeit, in: Meinel (Hrsg.), Verfassungsgerichtsbarkeit in der Bonner Republik (Fn. 39), S. 199. 
len teils jahrelange Diskussionen vorausgingen; intern - zumindest für das „Apothekenurteil“ ist das bekannt ${ }^{47}$ - zirkulierten sogar Sondervoten.

Damit werden die Akten zu einer wichtigen Quelle für das zeithistorische Verständnis der Entscheidungen. ${ }^{48}$ Darüber hinaus stellt sich die Frage, ob sie noch mehr leisten können. Dies macht erforderlich, das Verhältnis von juristischer Archivforschung und Rechtserkenntnis näher zu analysieren.

\section{Gerichtliches Archivmaterial und Rechtserkenntnis}

Versteht man Rechtserkenntnis als die Ermittlung des Regelungsinhalts einer Rechtsnorm, dann stellt sich zunächst die Frage nach dem normativen Stellenwert von Entscheidungen des Bundesverfassungsgerichts. Gerichtsentscheidungen und Gesetze sind auf unterschiedlichen Ebenen der Normenhierarchie angesiedelt. Gerichtliches Archivmaterial ist dementsprechend zunächst von Gesetzgebungsmaterialien zu unterscheiden. Um in Zweifelsfällen den Inhalt von Verfassung und Gesetzen zu ermitteln, können Materialien über deren Entstehung herangezogen werden. ${ }^{49}$

Die Unterscheidung gilt grundsätzlich auch für Entscheidungen des Bundesverfassungsgerichts. Zwar sind diese nach $\$ 31$ Abs. 1 BVerfGG mit umfassender Bindungswirkung ausgestattet. Formelle Gesetzeskraft entfaltet jedoch nach dessen Abs. 2 in Normenkontrollverfahren allein der Tenor der Entscheidung, der im Bundesgesetzblatt veröffentlicht wird. ${ }^{50}$ Es

47 Urteil vom 11.6.1958 - 1 BvR 596/56, BVerfGE 7, 377; Edition des internen Sondervotums bei Michl, JöR 68 (2020), 323.

48 Herbert, Was nützt eine Historisierung des Bundesverfassungsgerichts? Die Perspektive der Zeitgeschichte, in: Meinel (Hrsg.), Verfassungsgerichtsbarkeit in der Bonner Republik, (Fn. 39), S. 15.

49 Zur Rolle der Gesetzesmaterialien in der Rechtsanwendung Wischmeyer, JZ 2015, 957; Vogenauer, Die Auslegung von Gesetzen in England und auf dem Kontinent. Eine vergleichende Untersuchung der Rechtsprechung und ihrer historischen Grundlagen, 2 Bde., 2001; Frieling, Gesetzesmaterialien und Wille des Gesetzgebers. Fallgruppen verbindlicher Willensäußerungen, 2017.

50 Die Gesetzeskraft betrifft nur den Tenor, während die Bindungswirkung nach ständiger Rechtsprechung des BVerfG den Tenor und die ihn tragenden Gründe umfasst, s. bspw. BVerfG, Urteil vom 23.10.1951 - 2 BvG 1/51, BVerfGE 1, 14 (37); BVerfG, Beschluss vom 12.11.1997 - 1 BvR 479/92, BVerfGE 96, 375 (404). Kritisch dazu etwa Möllers, Legalität, Legitimität und Legitimation des Bundesverfassungsgerichts, in: Jestaedt/Lepsius/Möllers/Schönberger, Das entgrenzte Gericht. Eine kritische Bilanz nach sechzig Jahren Bundesverfassungsgericht, 2011, S. 281 (382-383). 
ist also die Anordnung, dass ein bestimmtes Gesetz nichtig ist, die normenhierarchisch Gesetzen entspricht. Um den Inhalt dieser Anordnung zu ermitteln, bedarf es keiner zusätzlichen Materialien; ihr objektiver Sinn ist - anders als bei Gesetzen - eindeutig.

Bedenken an der Relevanz gerichtlicher Archivakten können auch im Hinblick auf den durch das Beratungsgeheimnis geschützten Prozess richterlicher Rechtserkenntnis aufkommen. Juristische Archivforschung stellt so gesehen nicht nur Fragen für die heutige Rechtserkenntnis, sondern auch Fragen im Hinblick auf ihren Gegenstand selbst, also denjenigen richterlichen Rechtserkenntnisprozess, der die in den Blick genommene Entscheidung hervorbrachte. Ihr Entstehungsort ist das Beratungszimmer, dessen Türen strikt verriegelt sind. Nach $₫ 43 \mathrm{DRiG}^{51}$ hat der Richter „über den Hergang bei der Beratung und Abstimmung auch nach Beendigung seines Dienstverhältnisses zu schweigen". ${ }^{52}$ Die Bedeutung des Beratungsgeheimnisses, welches Ausdruck der richterlichen Unabhängigkeit ${ }^{53}$ ist, spiegelt sich in der 60 -jährigen Schutzfrist wider. ${ }^{54}$

Das Beratungsgeheimnis sichert darüber hinaus ab, dass die Entscheidungen selbst als eindeutige und mit Autorität verbundene Urteilssprüche nach außen treten. Daran anschließend könnte man argumentieren, dass ein Blick in die Archivmaterialien dazu geeignet ist, diese Autorität in Frage zu stellen, insbesondere, wenn sich zeigt, dass das Ergebnis der Entscheidung innerhalb eines kollegialen Spruchkörpers umstritten war und womöglich zeithistorischen und persönlichen Pfadabhängigkeiten unterlag.

Diese normativen Vorgaben scheinen eine klare Sprache zu sprechen. Mit Gesetzeskraft ist bei verfassungsgerichtlichen Urteilen nur die Entscheidungsformel ausgestattet. Das Beratungsgeheimnis schützt darüber hinaus den richterlichen Beratungsvorgang und damit indirekt auch die Autorität der Entscheidung selbst.

51 Anwendbarkeit auf das BVerfG nach $\$ 69$ DRiG.

52 Beim BVerfG bestehen durch die Zulässigkeit von Sondervoten sowie das Offenlegen der Stimmverhältnisse ( $\$ 30$ Abs. 2 BVerfGG) Einschränkungen.

53 Art. 97 Abs. 1 GG.

54 In der Gesetzesbegründung wird die lange Frist von 60 Jahren explizit mit „dem hohen Rang des Beratungsgeheimnisses“ (BT-Drucks. 17/13469, S. 1) begründet. 


\section{Gerichtliches Archivmaterial und Rechtsdogmatik}

Neben der Entscheidungsformel selbst enthalten die Entscheidungen des Bundesverfassungsgerichts eine Begründung. Die Entscheidungsgründe beinhalten ihrerseits die Argumente, die die Entscheidung stützen sollen. Das Gesetz statuiert eine Begründungspflicht ${ }^{55}$; der spezifische Begründungsstil des Bundesverfassungsgerichts hat sich darüber hinaus herausgebildet. Die Entscheidungen des Gerichts sind zumeist lang und durch Verweise auf die eigenen Vorläufer miteinander verbunden. ${ }^{56}$ Insbesondere die häufig sehr allgemeine Bezugnahme auf zuvor aufgestellte verfassungsrechtliche Maßstäbe kennzeichnet das rechtsdogmatische Selbstverständnis des Bundesverfassungsgerichts. ${ }^{57}$

Für die Kritik an der „maßstabsetzende[n] Gewalt“58 des Gerichts ist insbesondere Oliver Lepsius bekannt. Er fordert eine stärkere Historisierung und Kontextualisierung im Sinne einer Erweiterung der Dogmatik. ${ }^{59}$ Hierfür sprechen ihm zufolge vier Argumente: Erstens ermögliche Historisierung und Kontextualisierung, Pfadabhängigkeiten zu überdenken und zu korrigieren. Gerichtsentscheidungen müssten zweitens stärker in ihren historischen Zusammenhang eingeordnet werden. Dies gelte, drittens, insbesondere für ihren demokratischen Zeitbezug. Viertens mache dies der Rechtsprechungspluralismus in Europa erforderlich. ${ }^{60}$ Lepsius fordert daher eine stärkere Herausbildung von Fallgruppen, die sich an den zugrundeliegenden Sachverhaltskonstellationen orientieren. ${ }^{61}$

$55 \rrbracket 30$ Abs. 1 S. 2 BVerfGG. Zu den Ausnahmen zur Begründungspflicht s. Weber, Begründungsstil (Fn. 38), S. 64, zur historischen Entwicklung innerhalb Deutschlands s. ebd. S. 221-222.

56 Für eine Analyse der Länge und Struktur der Entscheidungen s. Weber, Begründungsstil (Fn. 38) S. 53-61, 127-145.

57 Zur Verfassungsrechtsdogmatik s. Jestaedt, Phänomen Bundesverfassungsgericht. Was das Gericht zu dem macht, was es ist, in: Jestaedt/Lepsius/Möllers/Schönberger, Das entgrenzte Gericht (Fn. 50), S. 77 (130-137).

58 Lepsius, Die maßstabsetzende Gewalt, in: Jestaedt/Lepsius/Möllers/Schönberger, Das entgrenzte Gericht (Fn. 50), S. 159.

59 Lepsius, JZ 2019, 793. Sein Anliegen ist ein „normatives Projekt“ im Sinne „einer die Dogmatik erweiternde[n] [...] Perspektive“, s. Lepsius, Über die Notwendigkeit der Historisierung und Kontextualisierung für die Verfassungsdogmatik, in: Meinel (Hrsg.), Verfassungsgerichtsbarkeit in der Bonner Republik (Fn.39), S. 119 (135). Ziel sei es, „immer mehr zu Tage tretende ernstliche Schwächen der Dogmatik" dadurch zu beheben (S. 137).

60 Ebd., S. 119-135.

61 Lepsius, Die maßstabsetzende Gewalt (Fn. 58), S. 159 (259-260). 
Historisierung und Kontextualisierung lässt sich durch gerichtliche Archivforschung operationalisieren. Sie dient damit auf unterschiedlicher Abstraktionsebene der Verfassungsrechtsdogmatik:

Auf einer abstrakten Ebene können Erkenntnisse über das Selbstverständnis des Bundesverfassungsgerichts als „Vorbedingung seriöser Verfassungsrechtsdogmatik“62 begriffen werden. Archivforschung muss sich dabei nicht darauf zurückziehen, ausschließlich „ein nuanciertes historisches Bild [...] zu zeichnen".63 Sie kann vielmehr dazu dienen, sich dem aufgrund des Beratungsgeheimnisses und der kollegialen Beratungskultur nicht öffentlichen Prozess der Meinungsbildung und Entscheidungsfindung anzunähern. ${ }^{64}$ Gegen die Bedenken in Bezug auf das Beratungsgeheimnis spricht dabei ein Vergleich mit der Zulässigkeit von Sondervoten und dem Mitteilen der Stimmenverhältnisse. Das Ziel von Archivforschung ist es nicht, spektakuläre Kehrtwenden im Verfassungsrecht zu vollziehen oder gar den „Schleier des Beratungsgeheimnisses $[$ [... $]$ vollständig [zu] lüften“65, sondern zum Verständnis der „Genese verfassungsgerichtlicher Entscheidungen" ${ }^{66}$ beizutragen.

Die Erkenntnisse der Archivforschung können auch konkrete argumentative Relevanz für die verfassungsrechtliche Dogmatik entfalten. Dafür ist detailgenau und fallgruppenspezifisch zu überprüfen, ob damalige Argumentationsmuster auf heutige Situationen übertragen werden können: Das Ergebnis ist keinesfalls eindeutig im Sinne einer historisierenden "Geschichtswerdung“ der Entscheidungen vorgezeichnet. Es kann sich ebenso zeigen, dass grundlegende verfassungsrechtliche Problemlagen den heutigen entsprechen. Der Nachvollzug der im Hintergrund stehenden

62 Meinel/Kram, JZ 2014, 913 (914).

63 Zitat nach Meinel, Einleitung, in: Meinel (Hrsg.), Verfassungsgerichtsbarkeit in der Bonner Republik (Fn. 39), S. 1 (9-10). Folgt man dem gesetzgeberischen Ziel der Änderung des BVerfGG, richtet sich der wissenschaftliche Auftrag primär an Historiker:innen. Die Gesetzesänderung ermöglicht danach „die Aufarbeitung der Geschichte des Bundesverfassungsgerichts und seiner Entscheidungen für das Verständnis und die Einordnung seiner Bedeutung für die Entwicklung einer stabilen Demokratie in der Bundesrepublik Deutschland“ (BT-Drucks. 17/13469, S. 1). Der Fokus liegt damit auf der zeithistorischen Erforschung des BVerfG als Institution.

64 Meinel, Einleitung (Fn. 63), S. 1 (9).

65 Michl, JöR 68 (2020), 324, der dies ebenso wie Roßbach als Ziel ablehnt, s. Roßbach, Weichenstellung und Vergangenheitsbewältigung (Fn. 44), S. 229 (231).

66 Meinel, Einleitung (Fn. 63), S. 1 (9). Ähnlich auch Michl, JöR 68 (2020), 324, der seine Analyse des "Sondervotums“ zum Apothekenurteil als „juristische Zeitgeschichtsforschung" verstanden wissen will (S. 376). 
Konfliktlagen und Argumentationslinien kann das verfassungsrechtliche Argumentationsrepertoire für heute erweitern.

Zusammenfassend verschafft gerichtliches Archivmaterial mehr Klarheit bei der Kontextualisierung von Entscheidungen. Damit kann Archivforschung zu einer informierten Dogmatik einen wichtigen Beitrag leisten. ${ }^{67}$

\section{Praktische Überlegungen zur Archivforschung}

Damit die Archivforschung verwertbare Ergebnisse produzieren kann, muss sie gleichzeitig ihre eigenen Grenzen vergegenwärtigen. Abschließend sollen daher einige praktische Überlegungen zur juristischen Archivforschung angesprochen werden.

\section{Selektivität von Archivforschung}

Das Material, das ins Archiv gelangt, ist in besonderem Maße selektiv. Zunächst handelt es sich bei der richterlichen Beratung um einen von Mündlichkeit und Kollegialität geprägten Prozess, der nicht notwendigerweise "Spuren in den Akten"68 hinterlässt. Sodann ist das Archiv auf die Kooperation mit der anbietenden Stelle angewiesen: Zwar besteht gesetzlich eine Anbietungspflicht, doch ist es schwer nachzuvollziehen, welche Akten womöglich erst gar nicht beim Archiv ankommen. ${ }^{69}$ Auch innerhalb des Archivs unterliegen die Akten einem Selektionsprozess: Die Archivar:innen entscheiden darüber, welche Akten „Unterlagen von bleibendem Wert" sind. Neben dieser Hürde ist mit der Aufnahme ins Archiv noch nichts über die Erschließung der Akten gesagt. Schließlich benötigt es Forscher:innen, die die Akten sichten, bewerten und der (Fach-) Öffentlichkeit präsentieren. All dies macht die Archivforschung zu einem schwierigen und zeitintensiven Unterfangen.

67 Ähnlich argumentieren auch Kram und Meinel. Sie sehen in der „historische[n] Abschichtung der Problemlagen“ die Möglichkeit für „die Rechtswissenschaft und die Auslegungspraxis, die heute mehr denn je benötigte Freiheit im Umgang mit der Verfassung und ihrer Deutungsgeschichte" zurückzugewinnen (Meinel) Kram, JZ 2014, 913 (915)).

68 Meinel, Einleitung (Fn. 63), S. 1 (9).

69 Für das BVerfG s. Hollmann, Die Akten des Bundesverfassungsgerichts im Bundesarchiv (Fn. 39), S. 97. 


\section{Europäisierung}

In der europäisierten Rechtsordnung dürfte es erforderlich sein, sich nicht allein mit der Genese bundesrepublikanischer Entscheidungen auseinanderzusetzen. Eine EU-Kompetenz für das Archivrecht besteht nicht. ${ }^{70}$ Die Regeln der verschiedenen Länder unterscheiden sich mitunter wesentlich. Teilweise existieren viel kürzere oder gar keine Schutzfristen. ${ }^{71}$ Für den französischen Conseil constitutionnel etwa wurde 2009 die Frist von 60 auf 25 Jahre verkürzt. ${ }^{72}$ Seit der Gesetzesänderung werden die wichtigsten Auszüge aus den Entscheidungsberatungen als „Les grandes délibérations du Conseil constitutionnel"73 herausgegeben - ein Editionsprojekt, das Vorbildwirkung haben könnte. Für den Gerichtshof der Europäischen Union sind die Akten nach einer Schutzfrist von 30 Jahren in den Historischen Archiven in Florenz verfügbar. ${ }^{74}$ Seit einigen Jahren werden auch diese Akten vermehrt wissenschaftlich untersucht. ${ }^{75}$

70 Dies liegt an der fehlenden Kompetenz für Kulturpolitik, s. Kaiser, Archiv und Recht (Fn. 18), S. 107 (108), auch zur völkerrechtlichen Dimension. S. außerdem den Bericht der Sachverständigengruppe über Fragen der Koordinierung im Archivwesen, Europäische Kommission, Generalsekretariat, Die Archive in der Europäischen Union, 1994.

71 Als Beispiele für Rechtsordnungen, die keine allgemeine Schutzfrist vorsehen, nennt Kaiser die US-amerikanische und in der EU die schwedische, italienische, niederländische, spanische und luxemburgische, s. Kaiser, Archiv und Recht (Fn. 18), S. 107 (110).

72 Loi organique $\mathrm{n}^{\circ}$ 2008-695 du 15 juillet 2008 relative aux archives du Conseil constitutionnel.

73 Mathieu/Machelon/Mélin-Soucramanien/Rousseau/Philippe (Hrsg.), Les grandes délibérations du Conseil constitutionnel 1958-1986, 2. Aufl. 2014.

74 Art. 1 (1) der VO (EWG, Euratom) Nr. 354/83 des Rates; Beschluss des Gerichtshofs der Europäischen Union vom 10.6.2014 über die Hinterlegung der historischen Archive des Gerichtshofs der Europäischen Union bei den historischen Archiven der Europäischen Union (Europäisches Hochschulinstitut) (2015/ C 406/02). S. dazu auch Audland, Journal of the Society of Archivists, 28 (1) 2007, 177.

75 In der Praxis können jegliche Seiten durch den Gerichtshof geschwärzt werden, die Rückschlüsse auf die geheimen Beratungen zulassen könnten, s. Nicola, Waiting for the Barbarians: Inside the Archive of the European Court of Justice, Washington College of Law Research Paper No. 2019-19, S. 5. Das Werk von Davies/ Nicola (Hrsg.), EU Law Stories, Contextual and Critical Histories of European Jurisprudence, 2017, enthält Analysen wichtiger Urteile, s. Rasmussen, Law Meets History. Interpreting the Van Gend En Loos Judgment, S. 103; Phelan, Goodbye to All That. Commission v. Luxembourg \& Belgium and European Community Law's Break with the Enforcement Mechanisms of General International Law, S. 121; 


\section{Digitalisierung}

Des Weiteren besteht eine Herausforderung in der Digitalisierung. Diese eröffnet zum einen neue Möglichkeiten für die archivalische Arbeit, insbesondere was die Zugänglichkeit von Materialien angeht. ${ }^{76}$ Zum anderen geht mit ihr auch einher, dass sich der archivalisch zu behandelnde Aktenbestand zukünftig immer weniger in seiner analogen Form präsentieren wird. Dies wirft Fragen auf, etwa im Hinblick auf die Auswahl von „Unterlagen von bleibendem Wert". ${ }^{\text {. }}$

Fritz, Tessili v. Dunlop 1976: The Political Background of Judicial Restraint, S. 357; Boerger/Davies, Imagining the Course of European Law? Parti Ecologiste 'Les Verts' v. Parliament as a Constitutional Milestone in EU Law, S. 83; Warlouzet, The Difficult Quest to Implement Cartel Control. Grundig-Consten (1966) and Philip Morris (1987), S. 261.

76 Häufig wird das Internet selbst als Archiv bezeichnet, s. Wirth, Archiv (Fn. 4), S. 17 (25). Zur Digitalisierung von Archiven s. auch Collin, Archive und Register. Verlorenes Wissen oder Wissensressource der Zukunft?, in: Schuppert/Voßkuhle (Hrsg.), Governance von und durch Wissen, 2008, S. 75; Collin, Die archivrechtliche Regulierung (Fn. 30), S. 209 (218-227). Aus archivwissenschaftlicher Sicht s. Metzing (Hrsg.), Digitale Archive - Ein neues Paradigma?, Beiträge des 4. Archivwissenschafltichen Kolloquiums der Archivschule Marburg, Veröffentlichungen der Archivschule Marburg Nr. 31, 2000. S. auch Naumann, Über die Nutzung digitaler Unterlagen in Archiven, in: Rehm/ Bickhoff (Hrsg.), Rechtsfragen (Fn. 33), S. 32.

77 Das 2017 in Kraft getretene neue BArchG dient nach der Gesetzbegründung da$\mathrm{zu}$, der Anpassung an den rasanten digitalen Fortschritt sowie an die Bedürfnisse der Informationsgesellschaft durch eine transparente Verwaltung Rechnung zu tragen, s. BT-Drucks. 18/9633, S. 27. Zur Digitalisierung und den Neuregelungen im BArchG als verspätet s. Berger, ZRP 2017, 15 und als teilweise unzureichend s. Berger, Öffentliche Archive und staatliches Wissen, 2019, insb. S. 341 ff.; positiver zur Neuregelung Sommerlatte, Das Bundesarchivgesetz - mehr Nutzer- und Wissenschaftsfreundlichkeit im Digitalen Zeitalter, in: Deiseroth/Weineke (Hrsg.), Zwischen Aufarbeitung und Geheimhaltung (Fn. 43), S. 47. Allgemein zur Neuregelung von 2017 s. Hollmann, Die Sicherung von Behörden- und Justizakten als staatliche Aufgabe. Anmerkungen zur Neufassung des Bundesarchivgesetzes, in: Deiseroth/Weinke (Hrsg.), Zwischen Aufarbeitung und Geheimhaltung (Fn. 43), S. 37. 
Juristische Archivforschung ist trotz der vorgetragenen normativen wie faktischen Einschränkungen ein wichtiger Baustein der heutigen Verfassungsrechtsdogmatik.

Angesichts der zunehmenden Digitalisierung wird der Blick auf die Regalreihen, die meterweise mit Akten gefüllt sind, allerdings immer mehr der Vergangenheit angehören. ${ }^{78}$ Was aber bleibt, ist der „Blick auf das offene Meer" ${ }^{679}$, den Archivforschung ermöglicht.

78 Auch virtuell bleibt das Abbild materialer Akten „in stilisierter Form“ vertraut, s. Vismann, Was weiß der Staat noch? (Fn. 1), S. 41 (42-43). Ein Bücherregal als Hintergrund ist auch bei den während der Corona-Pandemie häufigen, auch anlässlich der JTÖR Münster stattfindenden Videokonferenzen regelmäßig zu sehen. Es wird teilweise als Statussymbol beschrieben, s. bspw. Serrao, Das Bücherregal als neues Statussymbol, NZZ vom 9.4.2020, <https://www.nzz.ch/internatio $\mathrm{nal} /$ coronavirus-und-die-folgen-das-buecherregal-wird-zum-statussymbol-ld.15510 49>, abgerufen am 17.3.2021.

79 S. Zitat zu Beginn des Textes, Vismann, Was weiß der Staat noch? (Fn. 1), S. 41 (42). 
